\title{
ARTICLE Enduring effects of adolescent ketamine exposure on cocaine- and sucrose-induced reward in male and female C57BL/6 mice
}

Israel Garcia-Carachure ${ }^{1}$, Francisco J. Flores-Ramirez ${ }^{1}$, Samuel A. Castillo ${ }^{1}$, Anapaula Themann ${ }^{1}$, Miguel A. Arenivar ${ }^{1}$, Joshua Preciado-Piña ${ }^{1}$, Arturo R. Zavala $\mathbb{D}^{2}$, Mary Kay Lobo ${ }^{3}$ and Sergio D. Iñiguez $\mathbb{D}^{1}$

Ketamine has shown promising antidepressant efficacy for adolescent treatment-resistant depression. However, the potential enduring consequences of ketamine exposure have not been thoroughly evaluated. Thus, we examined if juvenile ketamine treatment results in long-lasting changes for the rewarding properties of sucrose and cocaine in adulthood, across three separate experiments. In Experiment 1, adolescent male and female C57BL/6 mice received ketamine (20 mg/kg) for 15 consecutive days (Postnatal Day [PD] 35-49). Twenty-one days later (PD70; adulthood) we examined their behavioral responsivity to sucrose (1\%) on a two-bottle choice design, or cocaine $(0,5,10 \mathrm{mg} / \mathrm{kg})$ using the conditioned place preference (CPP) test. We found that juvenile ketamine-pretreatment increased preference for sucrose and environments paired with cocaine in male, but not female, adult mice. This long-term outcome was not observed when male and female mice received ketamine as adults (PD70-84) and tested for sucrose and cocaine preference 21-days later (Experiment 2). Similarly, in Experiment 3, no long-lasting differences in these measures were observed when adolescent male mice were exposed to concomitant ketamine and social stressors (PD35-44), namely the social defeat or vicarious defeat stress paradigms-procedures that mediated a depression-related phenotype (along with a ketamine antidepressant-like response). Collectively, we demonstrate that in the absence of physical or psychological stress, adolescent ketamine exposure increases later life preference for the rewarding properties of sucrose and cocaine in a sex- and agespecific manner. As such, this preclinical work provides awareness for the potential long-term behavioral consequences associated with juvenile ketamine exposure.

Neuropsychopharmacology (2020) 45:1536-1544; https://doi.org/10.1038/s41386-020-0654-7

\section{INTRODUCTION}

Major depressive disorder (MDD) is a prevalent illness that negatively impacts the adolescent population [1]. Juveniles who develop MDD often display comorbid conduct and anxiety disorders [2], and up to $25 \%$ develop substance abuse [3, 4]. Even more concerning, $\sim 50 \%$ of young MDD-sufferers are unresponsive to traditional antidepressants, including the selective serotonin reuptake inhibitor fluoxetine-the only medication approved by the Food and Drug Administration for the treatment of depressive illnesses in the pediatric population. Consequently, alternative pharmaceutical treatments for MDD are actively being explored, particularly for youngsters, since the first incidence of depression is usually reported during adolescence, and suicidal ideation rises drastically during this time [5].

Clinical data indicates that ketamine (KET), a non-competitive $\mathrm{N}$ methyl-D-aspartate receptor antagonist, shows superior promise for the management of MDD symptomatology [6]. Specifically, repeated sub-anesthetic doses of KET in MDD patients result in a rapid and long-lasting antidepressant response [7-9]. Importantly, animal studies support these clinical findings in models of despair [10-12], providing a platform for future work to uncover the neurobiological factors that mediate its rapid therapeutic properties. These approaches, however, have focused on examining acute KET exposure in mostly adult populations [10, 13]. While the mechanisms underlying KET's antidepressant efficacy and duration have recently begun to be studied [6], its potential enduring neurobehavioral side effects are relatively unknown [14]. Aside from this, KET is a controlled substance that has abuse/ dependence potential [15], so it is surprising that KET is currently being administered to juvenile populations [16], when the brain is still undergoing development [17], and adolescents display higher predisposition for drug abuse [18]. Indeed, exposing children and adolescents to psychotropic agents is of concern because animal research shows that psychotropic pharmacological insults across ontogeny induce neurobehavioral consequences in later adulthood [19-24], particularly alterations in subsequent drug preference [25-30].

To date, researchers have focused on examining KET's anesthetic and analgesic effectiveness in pediatric populations [31, 32], with only few recent clinical studies examining its efficacy in treating adolescent mood-related illnesses [9, 16, 33, 34]. Although acute exposures to KET are being administered to treat $M D D$, it is imperative that the long-lasting consequences of chronic KET be assessed, given that both adult and adolescent patients on KET trials must return to the clinic for recurring treatment $[33,35]$. Indeed, animal work has already shown that

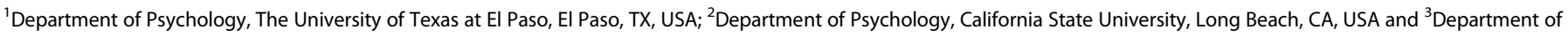
Anatomy and Neurobiology, University of Maryland School of Medicine, Baltimore, MD, USA

Correspondence: Sergio D. Iñiguez (sdiniguez@utep.edu)

These authors contributed equally: Israel Garcia-Carachure, Francisco J. Flores-Ramirez

Received: 3 October 2019 Revised: 11 February 2020 Accepted: 2 March 2020

Published online: 12 March 2020 
repeated KET exposure during adolescence induces long-term behavioral alterations to inescapable stressors [36]. Because vulnerability to stress influences drug-seeking behavior [37, 38], the goal of this investigation is to examine if adolescent KET treatment alters preference for reward-related stimuli in adulthood (i.e., sucrose and cocaine), and if so, whether or not this enduring effect is influenced by the age of KET exposure and/or social stress history.

\section{METHODS}

Animals

See Supplement for details. Experiments were conducted with approval of the Institutional Animal Care and Use Committee at The University of Texas at El Paso.

Drugs

Ketamine (KET)- and cocaine-hydrochloride were dissolved in sterile $0.9 \%$ saline (SAL) and administered via intraperitoneal injections at a volume of $2 \mathrm{ml} / \mathrm{kg}$. KET was purchased from Spectrum Chemicals (Gardena, CA), and cocaine from SigmaAldrich (St. Louis, MO).

\section{Experimental design}

To assess for enduring alterations in responsivity to reward-related stimuli, as a function of juvenile KET pretreatment, we conducted three experiments (Fig. 1). In Experiment 1 (Fig. 1a) adolescent male and female C57BL/6 mice received KET (0 [vehicle; VEH] or $20 \mathrm{mg} / \mathrm{kg}$ ) for 15 days (PD35-49). After KET or VEH exposure, male and female mice were left undisturbed in their home-cage for a 21-day washout period. At PD70 (i.e., adulthood), KET or VEH preexposed male and female mice were tested on the sucrose- or cocaine-preference tests. The age at the start and duration of KET exposure (PD35-49) was selected because it approximates

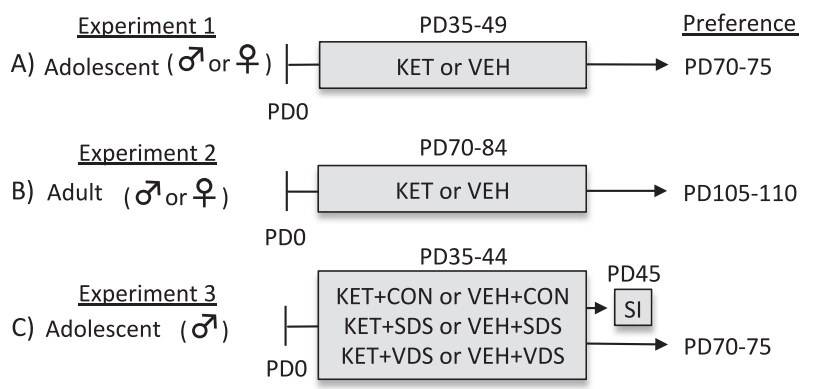

Fig. 1 Experimental design. a To evaluate if juvenile ketamine (KET) exposure mediates enduring alterations to reward-related stimuli in adulthood, adolescent (postnatal day [PD] 35) male and female C57BL/6 mice received KET $(20 \mathrm{mg} / \mathrm{kg}$ ) or saline (vehicle, VEH) for 15 consecutive days (PD35-49). Twenty-one days after the last KET exposure (PD70), mice were evaluated on the cocaine or sucrose preference tests (PD70-75). b To assess if KET pretreatment influenced preference for rewards as a function of age, adult (PD70) male and female mice were exposed to KET or VEH for 15 days (PD70-84), and after a 21-day washout period (PD105), they were evaluated on the cocaine or sucrose preference tests (PD105110). c Lastly, we examined how juvenile exposure to KET, in combination with either physical or psychological stress history would influence reward reactivity in adulthood. Specifically, adolescent male mice were exposed to either social defeat stress (SDS) or the vicarious defeat stress (VDS) paradigm for 10 consecutive days (PD35-44). Non-stressed control (CON) and stressed mice were administered with KET or VEH immediately after each SDS (KET+SDS) or VDS (KET+VDS) episode. Twenty-four hour post stress/KET treatment (PD45), one group of mice was evaluated on social interaction (SI) behavior. All other groups were evaluated on the cocaine or sucrose preference test once they reached adulthood (PD70-75). adolescence in humans [39]. Similarly, the KET dose $(20 \mathrm{mg} / \mathrm{kg} /$ day) was chosen because it yields significant effects on depression-related behavior in adolescents $[16,36]$. Because KET induces long-term alterations on despair-related behavior (i.e., forced-swim immobility) in both adolescent and adult male rodents [36], we conducted a separate set of similar experiments using PD70 male and female mice (as a positive control group for the age of KET exposure). Specifically, Experiment 2 (Fig. 1b) examined if 15-days of KET exposure in adult male and female mice (PD70-84) would alter sucrose and cocaine reactivity 21-days post treatment (PD105+). Lastly, because KET has distinct effects between healthy and depressed patients [40], in Experiment 3 (Fig. 1c) we evaluated how KET treatment along with social defeat stress (SDS) or vicarious defeat stress (VDS), during adolescence (PD35-44), would influence preference for cocaine or sucrose in adulthood (PD70+). To do this, we first validated the SDS and VDS paradigms as models of juvenile stress-induced behavior (i.e., reducing sociability; Fig. 4a) and antidepressant-efficacy (i.e., KET preventing stress-induced decreases in social behavior; Fig. 4c). We specifically selected SDS and VDS given that social stress is linked to the development of mood-related illnesses [41, 42]. Only male mice were used in Experiment 3 since juvenile KET preexposure increased reward-preference in adult male, but not female, mice (i.e., Experiments 1-2). Separate groups of animals were used across all experiments in order to avoid potential testing carryover effects (see Table S1).

Conditioned place preference

CPP was carried out as we have previously described [43] to assess the rewarding effects of cocaine [44, 45]. For specifics, see Supplement.

\section{Sucrose preference}

This single-day test consisted of a 2-bottle procedure [46] in which mice had the choice between consuming water or a $1 \%$ sucrose solution (details in Supplement).

Stress and social behavior

The juvenile SDS approach [47] with a witnessing component (i.e., VDS; 12, 48) was adopted to induce physical or psychological stress, as a function of KET exposure. Twenty-four hour after stress and/or KET exposure, one group of mice was tested on the social interaction test (details in Supplement).

Statistical analysis

Data were analyzed using ANOVA techniques, with KET-alone or KET-and-stress history, and cocaine post-treatment (between measures) as sources of variance. Tukey post hoc tests were used to examine all pairwise comparisons. Planned comparisons were also conducted to examine the hypothesis that KET pretreatment altered cocaine/sucrose-induced reward. Two-tailed Student's $t$ tests were used for analyses implicating two-group comparisons. Statistical significance was defined as $p \leq 0.05$. Data are presented as mean + SEM.

\section{RESULTS}

Adolescent KET exposure increases cocaine and sucrose preference in adult male mice

Figure $2 a, b$ displays the effects of male adolescent KET exposure (PD35-49) on cocaine (0, 5, or $10 \mathrm{mg} / \mathrm{kg}$ ) CPP in adulthood (PD70+; $N=60$ ). Specifically, a 2-way ANOVA (with KET-pretreatment and cocaine post-treatment as sources of variance) indicated that preference scores for the cocaine-paired side varied as a function of adolescent KET exposure (pretreatment main effect: $F_{1,54}=$ $13.54, p<0.05$ ) as well as cocaine exposure in adulthood (posttreatment main effect: $F_{2,54}=162.69, p<0.05$ ). Adolescent VEH $(n=10)$ or KET $(n=10)$ pretreatment did not influence preference 
A) Male Adolescent KET Pretreatment

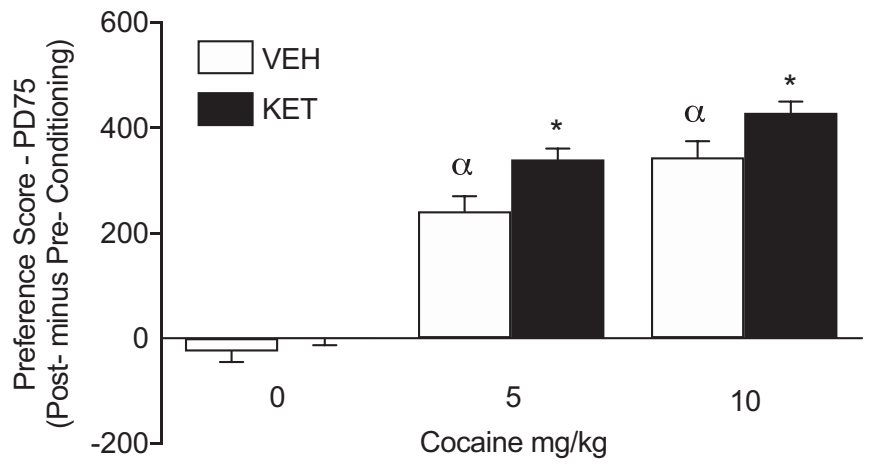

B)

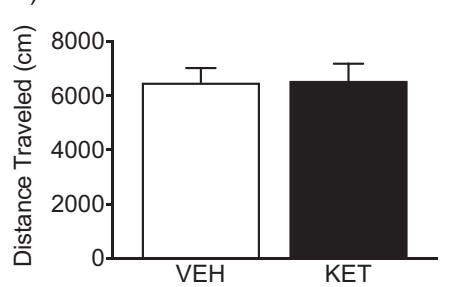

C)

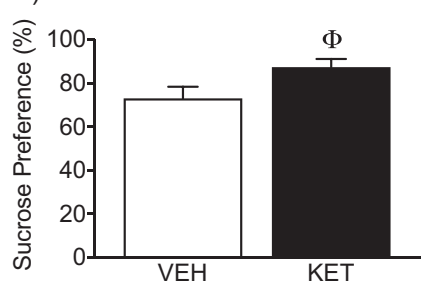

D)

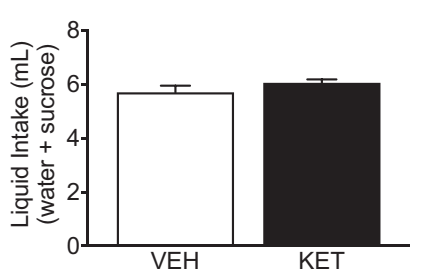

Fig. 2 Enduring effects of adolescent ketamine (KET) exposure for cocaine and sucrose preference in adult male mice. a Twenty-one days after adolescent KET exposure (postnatal day [PD] 70+), KET-pretreated mice displayed an enhanced preference to 5 and $10 \mathrm{mg} / \mathrm{kg}$ cocaine, when compared to saline (VEH)-pretreated control mice receiving the same doses of cocaine $(n=10$ per experimental group; $p<0.05)$. Asterisk represent within cocaine-dose group comparison $(p<0.05)$. Alpha represent significantly different when compared to age-matched controls conditioned to saline $(p<0.05)$. $\mathbf{b}$ No differences in distance traveled $(\mathrm{cm})$ were evident during the preconditioning phase, between the experimental groups. c Adolescent KET history increased preference for a $1 \%$ sucrose solution, three weeks after KET exposure $(n=10$ per group; $\left.{ }^{\Phi} p<0.05\right)$. d No differences in total liquid intake were observed between the experimental groups $(p>0.05)$.

for any of the compartments of the apparatus, when mice were conditioned to SAL $(p>0.05)$. On the other hand, VEH-pretreated mice conditioned to $5(n=10)$ or $10 \mathrm{mg} / \mathrm{kg}(\mathrm{n}=10)$ cocaine displayed reliable conditioning for the cocaine-paired compartment, when compared to control (VEH-pretreated/SAL-conditioned) mice $\left({ }^{a} p<0.05\right)$. KET-pretreated mice also displayed reliable conditioning to the compartment paired with $5(n=10)$ or $10(n=10) \mathrm{mg} / \mathrm{kg}$ cocaine, when compared to KET-pretreated/ SAL-conditioned mice $(p<0.05$, respectively). Interestingly, KETpretreated mice conditioned to 5 or $10 \mathrm{mg} / \mathrm{kg}$ cocaine, displayed higher preference scores for the drug-paired compartment $\left({ }^{*} p<\right.$ 0.05 , respectively) when compared to VEH-pretreated mice receiving the same dose of cocaine in adulthood (i.e., within dose comparison). Importantly, there were no differences in distance traveled $(\mathrm{cm})$, as a function of adolescent KET or VEH pretreatment, during the preconditioning phase $(p>0.05$; Fig. $2 b)$; indicating no differences in general locomotor activity between the groups.

Figure $2 c$ shows the enduring effects of male adolescent KET exposure (PD35-49) on sucrose preference in adulthood (PD70+). A $t$ test indicated that adult male mice with juvenile KET history $(n=10)$ displayed a significantly higher preference for a $1 \%$ sucrose solution when compared to VEH-pretreated $(n=10)$ controls $\left({ }^{\Phi} t_{18}=2.20, p<0.05\right)$. No differences in total liquid intake (water+sucrose) were noted ( $p>0.05$; Fig. $2 d$ ).

Adult KET exposure in male mice does not influence cocaine and sucrose preference later in life

Figure $3 a$, b displays the enduring effects of adult KET pretreatment (PD70-84) on cocaine CPP, in male mice (PD105+). Here, a 2way ANOVA (with KET-pretreatment and cocaine post-treatment as sources of variance) showed that preference scores were influenced by cocaine exposure (post-treatment main effect: $F_{2,54}$ $=203.17, p<0.05)$. Specifically, KET $(n=10)$ or VEH $(n=10)$ pretreatment did not influence preference for any of the compartments conditioned to SAL $(p>0.05)$. In contrast, VEH- or KET-pretreated mice conditioned to $5(n=10)$ or $10 \mathrm{mg} / \mathrm{kg}(n=$ 10) cocaine, displayed reliable preference scores, when compared to VEH-pretreated/SAL-conditioned mice $\left({ }^{\varsigma} p<0.05\right)$. Similarly, KETpretreated male mice displayed a preference for environments paired to $5(n=10)$ or $10 \mathrm{mg} / \mathrm{kg}$ cocaine $(n=10)$, when compared to KET-pretreated/SAL-conditioned mice $(p<0.05$, respectively). Planned comparisons indicated that no differences in preference scores were evident between SAL- and KET-pretreated mice receiving the same doses of cocaine ( $p>0.05$, respectively). No differences in distance traveled $(\mathrm{cm})$ during the preconditioning phase, as a function of KET pretreatment, were observed between the groups ( $p>0.05$; Fig. $3 \mathrm{~b}$ ).

Figure $3 c$ shows that adult KET pretreatment (PD70-84), in male mice, did not alter preference for a $1 \%$ sucrose solution $(p>0.05$; $n=5$ per group), or total liquid intake ( $p>0.05$; Fig. 3d), 21-days later (PD105+).

Female KET exposure, during adolescence or adulthood, does not alter cocaine or sucrose preference in later life

The long-term effects of female juvenile (PD35-49) or adult (PD7084) KET history, on cocaine and sucrose preference, are shown in Figs. S1 and S2, respectively. For specific data analyses see Supplemental and Table S2. Briefly, while cocaine mediated CPP across the groups (Figs. S1A and S2A; $n=10$ /group), there were no differences in preference magnitude between VEH- or KETpretreated female mice receiving the same cocaine dose (independent of age of KET pre-exposure). No differences in locomotor activity, during the preconditioning phase, were noted between the groups (Figs. S1B and S2B). Similarly, female KET preexposure (during adolescence or adulthood) did not influence sucrose preference or total liquid intake, later in life (Fig. S1C, D, $n=9$ /group; and Fig. S2C, D, $n=10 /$ group). 
A)

Male Adult KET Preteatment
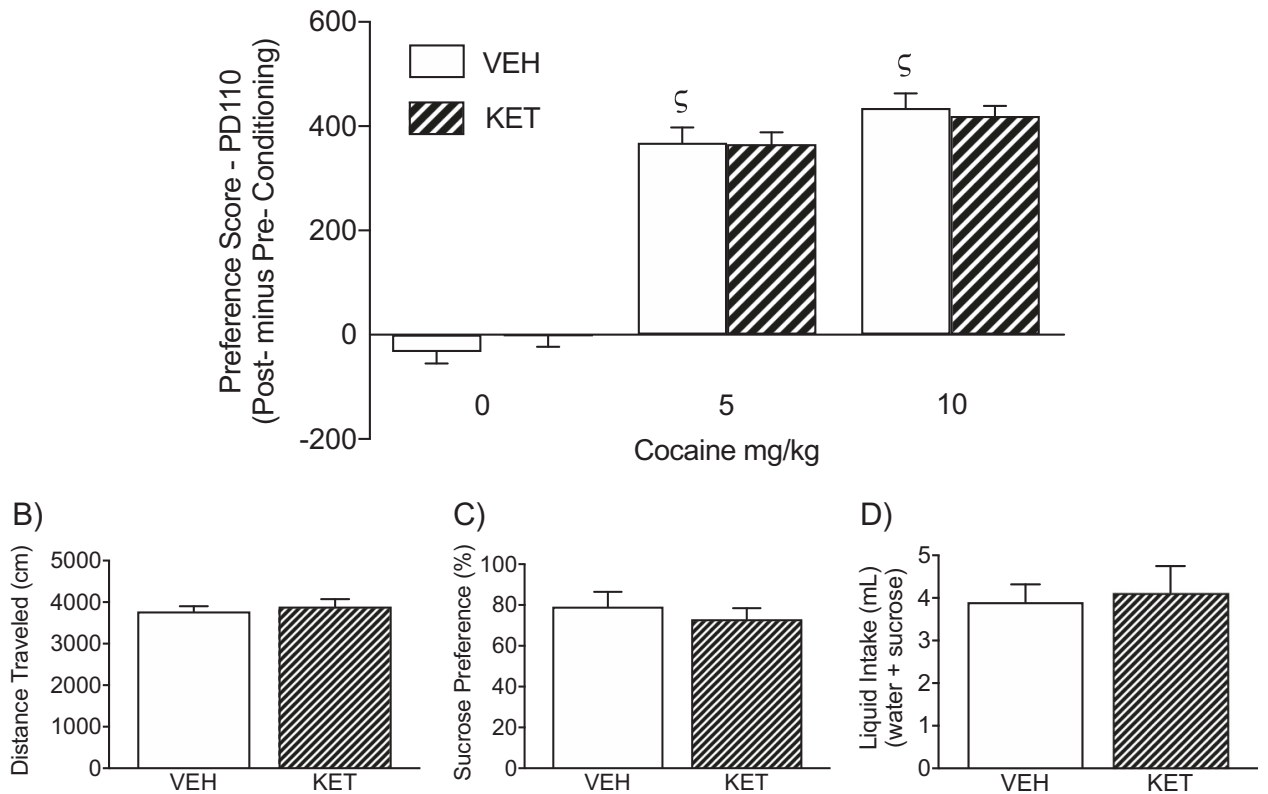

Fig. 3 Enduring effects of adult ketamine (KET) or saline (VEH) exposure (postnatal day [PD] 70-84) on cocaine and sucrose preference in male mice. a Twenty-one days after adult KET exposure (PD105+), VEH- and KET-pretreated mice conditioned to 5 or $10 \mathrm{mg} / \mathrm{kg}$ cocaine displayed reliable preference scores to the cocaine-paired side, when compared to VEH-pretreated/SAL-conditioned mice ( ${ }^{\varsigma} p<0.05 ; n=10$ per experimental condition). No differences in preference scores were evident between VEH- and KET-pretreated mice receiving the same doses of cocaine $(p>0.05$, respectively). b No differences in distance traveled $(\mathrm{cm})$ were evident during preconditioning phase, between the experimental groups. c Adult KET pre-exposure did not influence preference for a 1\% sucrose solution three weeks after KET treatment $(n=5$ per group; $p<0.05)$. d No differences in total liquid intake were observed between the KET or VEH pretreated groups $(p>0.05)$.

Establishing depression-related behavior and KET antidepressantefficacy in juvenile male mice

Figure 4 shows the effects of social stress (VDS or SDS)-alone, or along with KET treatment, on social behavior in adolescent male mice. A one-way ANOVA (within vehicle pretreated mice) indicated that social interaction ratios varied as a function of stress exposure $\left(F_{2,27}=12.15, p<0.05\right)$. Tukey post hoc comparisons indicated that, when compared to non-stressed controls $(\mathrm{CON}, n=10)$, mice exposed to VDS $(n=10)$ or SDS $(n=10)$ displayed significantly lower social interaction ratios (Fig. 4a)-a depression-related phenotype $[48,49]$. Conversely, in Fig. 4c, a one-way ANOVA (within KET exposed mice) indicated that social interaction ratios did not differ as a function of stress $[p>0.05$; CON $(n=10)$, VDS $(n=10)$, or SDS $(n=10)]$, showing that KET prevents adolescent stress-induced decreases in sociability, in a similar fashion as adult rodents $[12,50]$. Importantly, no differences in locomotor activity were noted as a function of stress type, independent of VEH ( $p>0.05$, Fig. 4b) or KET ( $p>0.05$, Fig. 4d) pretreatment.

Adolescent KET+VDS or KET+SDS history does not alter cocaine or sucrose preference in adult male mice

Figure $5 \mathrm{a}$ shows the long-term effects of concomitant juvenile KET (or VEH) and stress (KET+SDS or VEH+SDS; KET+VDS or VEH+VDS) pre-exposure (PD35-44) on cocaine CPP in male mice (PD70+). A three-way ANOVA (with KET and/or stress pre-exposure, and cocaine post-treatment, as sources of variance) revealed that preference scores varied as a function of cocaine (post-treatment main effect: $F_{2,155}=76.45, p<0.05$ ) and a marginal stress $\times \mathrm{KET} \times$ cocaine interaction $\left(F_{4,155}=2.12, p=0.08\right)$. Neither stress history (CON, SDS, or VDS), with or without KET pre-exposure, influenced preference scores for the compartment conditioned to SAL ( $n=8-10$, per group; $p>0.05$ ). In contrast, VEH+CON preexposed mice conditioned to 5 or $10 \mathrm{mg} / \mathrm{kg}$ cocaine displayed a significant preference for the cocaine paired-compartment, when compared to VEH+CON-pretreated/SAL-conditioned mice $\left({ }^{a} p<0.05\right)$. Similarly, mice exposed to stress alone (VEH+VDS or $\mathrm{VEH}+\mathrm{SDS}$ ) or in combination with KET (KET+CON, KET+VDS, or KET +SDS) displayed significant preference for the compartment paired with 5 or $10 \mathrm{mg} / \mathrm{kg}$ cocaine, when compared to their respective SAL-conditioned groups $(p<0.05)$. Unexpectedly, VEH+VDS mice displayed enhanced conditioning scores to 5 and $10 \mathrm{mg} / \mathrm{kg}$ cocaine $\left({ }^{*} p<0.05\right.$, respectively), when compared to $\mathrm{VEH}+\mathrm{CONs}$ receiving the same doses of cocaine. Furthermore, KET-pretreated mice without stress history $(\mathrm{KET}+\mathrm{CON})$ also displayed enhanced CPP scores to 5 and $10\left({ }^{*} p \leq 0.05\right) \mathrm{mg} / \mathrm{kg}$ cocaine, when compared to $\mathrm{VEH}+\mathrm{CON}$ mice receiving the same cocaine doses-replicating the findings from Experiment 1 (Fig. 2a). Lastly, a three-way ANOVA (with stress, KET, and cocaine as sources of variance) indicated that distance traveled, during the preconditioning phase, varied as a function of KET history $\left({ }^{\beta} \mathrm{KET}\right.$ pretreatment main effect: $F_{1,167}=$ $12.41, p<0.05)$. Specifically, mice receiving KET (KET+CON, KET +VDS, KET+SDS) displayed significantly lower distance traveled (Fig. 5b), when compared to VEH-pretreated groups (VEH+CON, $\mathrm{VEH}+\mathrm{VDS}, \mathrm{VEH}+\mathrm{SDS}$ ).

Figure $5 c$, $d$ shows the long-lasting effects of adolescent KET and stress history (PD35-44) on sucrose preference in adulthood $($ PD70+). A two-way ANOVA (with stress and KET as sources of variance) indicated that juvenile stress exposure altered sucrose preference in adulthood ( ${ }^{x}$ stress main effect: $F_{2,54}=18.33, p<$ 0.05). Specifically, mice with stress history (VEH+VDS, VEH+SDS, $\mathrm{KET}+\mathrm{VDS}, \mathrm{KET}+\mathrm{SDS}$ ) displayed significantly lower preferences for sucrose, when compared to non-stressed groups (VEH+CON, KET + CON), in adulthood. Lastly, a two-way ANOVA, with stress and KET history as sources of variance, indicated that total liquid intake ([water+sucrose]/weight) varied as a function of KET pretreatment $\left({ }^{\beta}\right.$ KET-pretreatment main effect: $\left.F_{1,54}=10.92, p<0.05\right)$. Specifically, independent of stress pre-exposure (VEH+CON, VEH+VDS, $\mathrm{VEH}+\mathrm{SDS}$ ), KET pretreated animals (KET+CON, KET+VDS, KET + SDS) displayed lower total liquid intake $(M=0.15 \mathrm{~mL} / \mathrm{g})$ when 
Establishing depression-related behavior and KET antidepressant-efficacy in juvenile male mice

A)

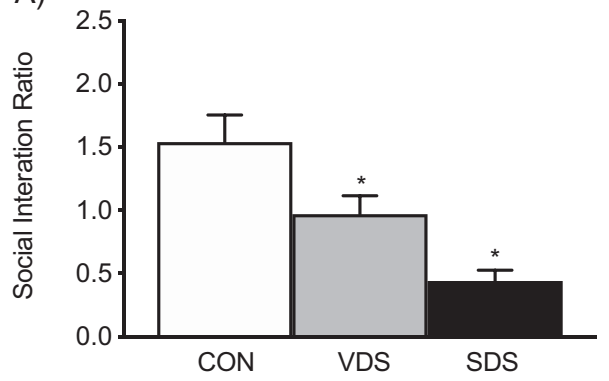

C)

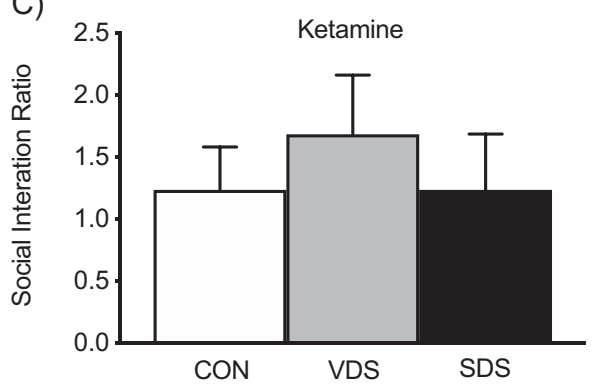

B)

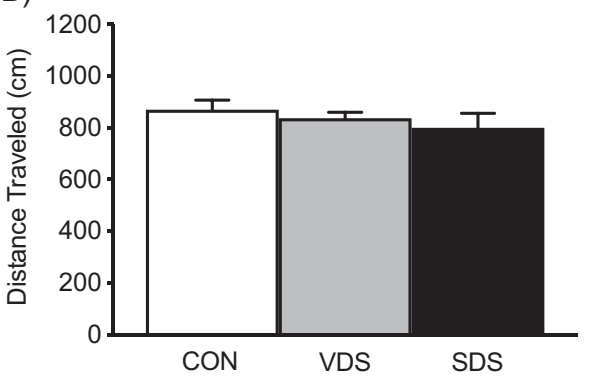

D)

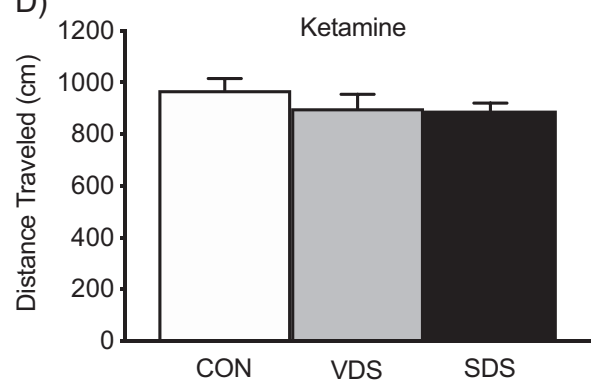

Fig. 4 Ketamine mediates prophylactic effects on depression-related behavior in adolescent male mice (postnatal day 45). a When compared to CON's [non-stressed], vicarious defeat stress (VDS/vehicle)- and social defeat stress (SDS/vehicle)-exposed mice displayed decreases in social interaction ratios, b without altering locomotor activity $(p>0.05)$. c Conversely, ketamine exposure (after each defeat episode) prevented the stress-induced reductions of social behavior across the groups $(p>0.05)$. $\mathbf{d}$ No differences in locomotor activity (distance traveled) were noted between the stress and ketamine-pretreated groups ( $n=10$ per group). * $p<0.05$ when compared to CON.

compared to mice pre-exposed to VEH $(M=0.20 \mathrm{~mL} / \mathrm{g})$. Lastly, Fig. S3 demonstrates the long-lasting effects of adolescent KET and stress history (PD35-44) on body weight in adulthood (PD70). A two-way ANOVA indicated that body weight did not differ between the experimental groups, as a function of KET-pretreatment, stress-type, or their interaction ( $p>0.05$, respectively).

\section{DISCUSSION}

KET is currently being dispensed to juvenile populations for the management of MDD, given its rapid antidepressant therapeutic effects $[9,16,33,34]$. Such off-label pharmacological approach is concerning, since KET is a controlled substance with abuse potential [51] and could result in unexpected long-lasting side effects $[36,52]$. Therefore, the purpose of this investigation was to assess if adolescent KET exposure alters preference for rewardrelated stimuli in adulthood; a preclinical approach that could inform about KET-induced predisposition to drug use-vulnerability in later life [29, 44,53]. We report that juvenile KET exposure increases preference for the rewarding properties of cocaine and sucrose in male, but not female, adult mice. Moreover, this enduring male-specific effect is dependent on the age of KET preexposure (i.e., adolescence) as well as the absence of physical (SDS) or psychological (VDS) stress history.

CPP is a behavioral paradigm that is commonly implemented to evaluate the rewarding properties of drugs of abuse [29, 54]. Adopting Pavlovian learning principles, CPP is used to measure increases in the incentive motivation value of rewarding stimuli, as they become associated with a specific environment and thus it is considered a model of reward-seeking behavior [44]. Accordingly, implementing this well-established approach, we found that independent of juvenile KET pretreatment, adult male and female mice (PD75+) displayed a preference for the cocaine-paired compartment (Fig. 2a and S1A, respectively). Interestingly, within the male groups (Fig. 2a), adult mice (PD75+) pre-exposed to KET during adolescence (PD35-49) displayed enhanced CPP magnitude, when compared to corresponding controls receiving the same cocaine doses. This suggests that juvenile KET history increases reactivity to the rewarding effects of cocaine in adulthood [44]. In line with this, KET-pretreated adult male mice displayed higher preference for sucrose (Fig. 2c), when evaluated on a single-day two-bottle test. Conversely, no alterations to either reward-related stimuli were observed in adult female mice pretreated with KET during adolescence, when compared to respective controls (Fig. S1). Importantly, KET pretreatment did not alter general locomotor activity (Fig. 2b, S1B, and 52) or total liquid intake in neither male nor female mice (Fig. 2d and S1D, respectively). These findings unmask an enduring KET-induced increase in preference for cocaine and sucrose in males specifically.

Previous work indicates that repeated exposure to KET, during adolescence or adulthood, alters sensitivity to inescapable stressors two-months after drug pretreatment [36], highlighting a long-lasting KET-induced effect. Therefore, we exposed adult (PD70) male and female mice to KET for the same amount of time (15-days) and evaluated their preference for cocaine or sucrose at a similar post-treatment interval (21-days). As expected, PD105+ male and female mice acquired cocaine CPP (Fig. 3 and Fig. S2, respectively). However, in this case, neither PD105+ male nor female mice (pre-exposed to KET from PD70-84) displayed enhanced CPP scores when compared to respective VEHcontrols administered with the same doses of cocaine. Similarly, no differences in sucrose preference were noted as a function of adult KET pre-exposure in either male or female mice (Fig. $3 \mathrm{c}$ and Fig. S2C). Together, these sex- (compare Fig. 2a and S1A) and agedependent (compare Figs. $2 a$ and $3 a$ ) effects confirm that adolescence is a developmental window of vulnerability (to KET exposure) resulting in long-lasting alterations to reward reactivity in males, but not females. Furthermore, these data support previous work demonstrating that juvenile psychotropic drug exposure leads to enduring neurobiological changes that are persistent in adulthood [26, 27], yet, here we expand these 
A)

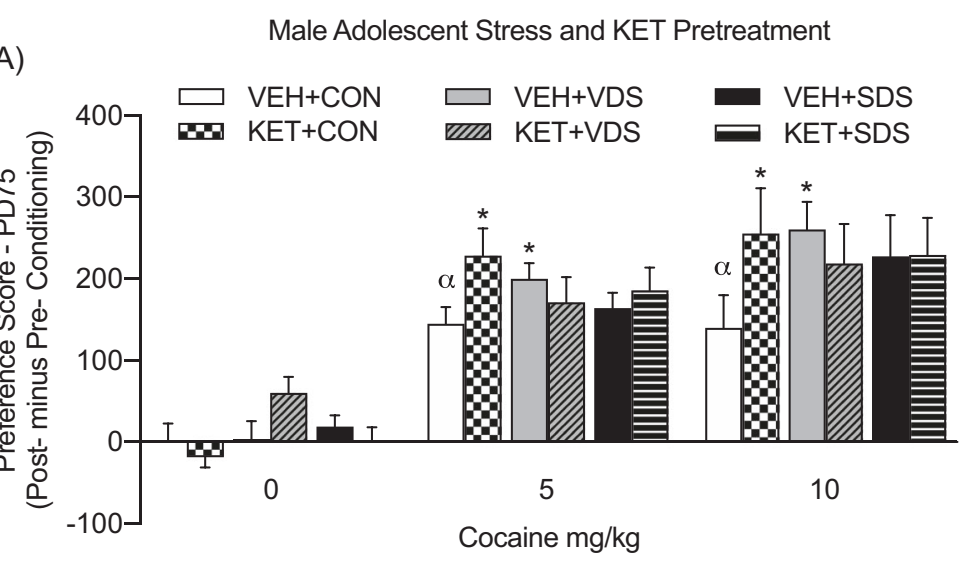

B)

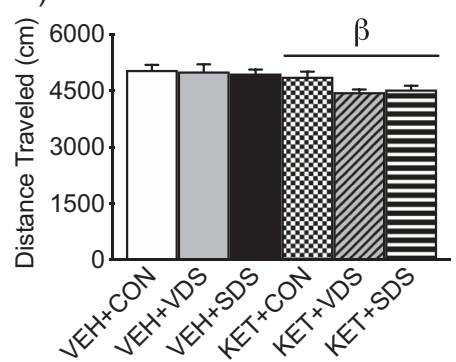

C)

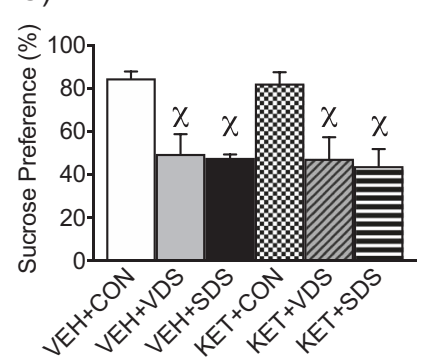

D)

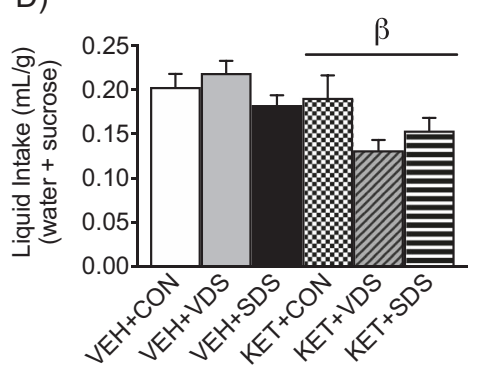

Fig. 5 Enduring effects of male juvenile exposure to concomitant ketamine (KET)-and-stress [social defeat stress (SDS), vicarious defeats stress (VDS), or no-stress control (CON)] on cocaine and sucrose preference in adulthood. a Neither KET or stress combination treatment ( $n=8-10$ per group) influenced preference scores for the compartment conditioned to saline (i.e., $0 \mathrm{mg} / \mathrm{kg}$ cocaine; $p>0.05$ ). In contrast, all experimental male mice displayed reliable conditioning to 5 and $10 \mathrm{mg} / \mathrm{kg}$ cocaine, when compared to respective groups conditioned to saline (cocaine main effect, $p<0.05$ ). Furthermore, adult mice pre-exposed to saline-vehicle (VEH) and VDS during adolescence, showed an enhanced preference to 5 and $10 \mathrm{mg} / \mathrm{kg}$ cocaine, when compared to $\mathrm{VEH}+\mathrm{CON}$ mice receiving the same doses $\left({ }^{*} p<0.05\right.$, respectively). Similarly, adult mice pre-exposed to KET+CON showed a higher preference score to 5 and $10\left({ }^{*} p \leq 0.05\right) \mathrm{mg} / \mathrm{kg}$ cocaine, when compared to $\mathrm{VEH}+\mathrm{CON}$ mice receiving the same doses of cocaine. b KET-pretreatment, independent of stress history, resulted in lower distance traveled during the preconditioning phase (main effect of stress, ${ }^{\beta} p<0.05$ ). c Adolescent stress history (VDS or SDS), independent of KET or VEH pretreatment, resulted in decreased preference for a $1 \%$ sucrose solution in adulthood (main effect of stress; $x_{p}<0.05$ ). d KET-pretreatment, independent of stress history, resulted in decreased liquid intake in adulthood (main effect of KET-pretreatment, ${ }^{\beta} p<0.05$ ).

findings to KET-induced alterations for enhanced cocaine and sucrose reward in a sex- and age-specific manner.

Clinical studies report that KET mediates differential effects between normal human volunteers and MDD patients [40]. Thus, we evaluated if adolescent KET-pretreatment along with social stress history would influence reward preference in adulthood. We selected SDS and VDS given that these physical and psychological forms of stress reduce social behavior-a depression-related phenotype frequently reported in adult rodents $[47,49,55]$ that is rescued by chronic administration of traditional antidepressants [56] and acute KET exposure [12, 50]. Adopting these stress paradigms, we forced adolescent male C57BL/6 mice to intrude the territorialized cage of a resident aggressor CD1 mouse, resulting in an agonistic physical encounter (SDS), while an observer adolescent C57BL/6 mouse vicariously experienced the stress about from the safety of an adjacent compartment (VDS), once per day for 10 consecutive days $[12,48,57]$. After each stress episode, SDS, VDS, and non-stressed (CON) mice were administered KET or VEH (Fig. 1c). To validate this approach as a model for the study of adolescent stress-induced depression, one subgroup of mice was evaluated on social behavior the following day (PD45). We found that adolescent mice exposed to SDS [47] and VDS [55] displayed lower social interaction ratios, when compared to CONs (Fig. 4a). Importantly, we show for the first time that exposure to KET prevents stress-induced decreases of social behavior in juvenile mice (Fig. 4c), establishing pharmacological validity for the study of depressive- and antidepressant-like efficacy in the juvenile SDS/VDS model [57]. Consequently, after additional groups of ketamine-and-stress pre-exposed mice reached adulthood (PD70), they were evaluated for cocaine and sucrose reward; matching the age of behavioral testing of stressnaive animals pretreated with KET or VEH in our initial experiment (Fig. 1a). Here, PD75 mice with adolescent history of psychological stress (VEH+VDS), or KET+CON, displayed higher preference scores to cocaine, when compared to non-stressed controls (VEH $+\mathrm{CON})$ exposed to the same doses of the stimulant $(p<0.05)$. This indicates that juvenile exposure to VDS-, or KET-alone, mediates long-term increases for cocaine seeking behavior, in a similar fashion as early-life exposure to stress or pharmacological insults $[26,27,58,59]$. Notably, while previous work suggests that SDSalone mediates an enduring increase for cocaine CPP [60], here, $\mathrm{VEH}+\mathrm{SDS}$ pre-exposed mice did not differ from the VEH+CON group receiving the same cocaine dose. This discrepancy is likely due to differences in mouse strain (OF1 vs C57BL/6), stress protocol, and dose of cocaine used ( $1 \mathrm{vs} 5-10 \mathrm{mg} / \mathrm{kg}$ ) between the studies. Interestingly, the cocaine-induced CPP scores in animals exposed to concomitant KET and stress (KET+VDS or KET+SDS) did not differ in magnitude from saline/non-stressed control mice $(\mathrm{VEH}+\mathrm{CON})$. Hence, revealing that adolescent exposure to combined stress and KET-modeling antidepressant treatment for MDD-does not result in long-lasting changes to rewardrelated stimuli.

When we evaluated reactivity to sucrose, as a function of juvenile stress and/or KET pre-exposure, we found that independent of KET history, stress (VDS and SDS)-exposed mice displayed reduced preference for this natural reward-replicating a well 
established long-term SDS- and VDS-induced anhedonia-like phenotype $[48,61,62]$ that was not rescued by KET pretreatment during adolescence (Fig. $5 \mathrm{c}$ ). This was anticipated, given that both animal models [50], as well as MDD patients [7-9], must receive repeated KET infusions to ameliorate depression-related phenotypes. However, unlike Experiment 1, no differences in sucrose preference were noted between $\mathrm{KET}+\mathrm{CON}$ and $\mathrm{VEH}+\mathrm{CON}$ groups. This inconsistent finding across experiments (see Figs. 2c and $5 \mathrm{C}$ ) is likely due to differences in the number of KET/VEH injections received during adolescence (15 vs 10), as well as housing conditions during the drug washout period (grouped vs segregated). Unexpectedly, KET-history decreased liquid intake (Fig. 5d) and locomotor activity (Fig. 5b); yet, such factors were not likely to influence responses to reward-related stimuli, since no differences in body weight were noted between the groups (Fig. S3), and total liquid was normalized to body weight for the sucrose preference test (Fig. $5 \mathrm{~d}$ ). Of course, future work will be needed to evaluate the consequences of adolescent KET exposure on these physiological measures, particularly, since decreased liquid intake could be an indication of oligodipsia, a syndrome correlated with dissociative experiences [63, 64].

The neurobiological mechanisms by which early-life KET exposure increases reward-related preference in adulthood have not been evaluated. During adolescence, KET increases locomotor activity in a sex-specific manner [65], with males displaying a delayed response when compared to females. Interestingly, monoamine systems underlie, at least in part, this age and sexspecific KET-induced pattern of locomotor activation [66], which in turn, could provide insight to the male-specific consequences of KET on reward-related stimuli in adulthood (or enduring female resiliency to KET). For example, acute KET administration (i.e., $10-50 \mathrm{mg} / \mathrm{kg}$ ) increases dopamine levels in the prefrontal cortex, nucleus accumbens, and striatum, while increasing the firing of dopamine neurons within the ventral tegmental area; [67] circuitry involved in reward-related behavior. Thus, repeated exposure to KET during adolescence may result in long-lasting and sex-specific changes within the dopamine system, altering reward-seeking behavior in adulthood $[68,69]$. Supporting this hypothesis, human recreational KET users display increases in dopamine receptor 1 (DR1) within the medial prefrontal cortex [70], highlighting a potential neurobiological mechanism by which repeated early-life exposure to KET (without stress history) may increase preference for cocaine in later life. Indeed, the expression of cocaine CPP is modulated by DR1 antagonism within this brain region in male rodents [69], so it is possible that juvenile KET history may result in altered DR1 expression in a sex-specific manner, as it has been shown in other brain regions [71]. Conversely, the absence of long-lived increases in cocaine or sucrose preference, post juvenile exposure to concomitant stress-and-KET, could be attributed to the individual and oppositional effects between KET and stress on brain derived neurotropic factor (BDNF)-related signaling [10, 72], a molecular mechanism that modulates behavioral responses to cocaine and that is also implicated in MDD [73-75].

In rodent models of physical and/or psychological stress, KET acutely reverses or prevents the development of depressionrelated phenotypes (Fig. 4, and [12, 50, 76]. However, to our knowledge, the enduring effects of repeated juvenile KET exposure on cocaine- and sucrose-preference, with or without social stress history, have not been evaluated. Here we report, for the first time, that adolescent KET history in stress-naïve mice increases preference for cocaine and sucrose in adult male, but not female, C57BL/6 mice; suggesting a potential enduring malespecific increase in risk for drug use-vulnerability. However, juvenile KET exposure, along with physical or psychological stress, does not induce long-lasting alterations to drug or natural rewards. Collectively, this preclinical model provides first line evidence about the safety of chronic KET exposure during adolescence, post recreational use, or when administered as a treatment for stress-induced affect-related illnesses.

\section{FUNDING AND DISCLOSURE}

No financial or non-financial interests in relation to the work described in this manuscript is declared by the authors. This project was supported by the National Institute of General Medical Sciences (NIGMS-1SC3GM130467, to SDI). Student co-authors were financially supported to attain basic research experience/training per their respective program objectives. Specifically, FJF-R was supported by a Summer Research Grant from The Graduate School at UTEP. IG-C and SAC were funded by the LSAMP (Louis Stokes Alliance for Minority Participation) Bridge to Doctorate support program. MAA was supported by the SMART-MiND (Summer Mentoring and Research Training: Methods in Neuroscience of Drug-abuse) program. JP-P was supported by the BUILD (Building Infrastructure Leading to Diversity) Scholars program at UTEP. The authors declare no conflict of interest.

\section{ACKNOWLEDGEMENTS}

We would like to thank Omar Lira, Minerva Rodriguez, and Adolfo Andazola-Carmona for technical assistance.

\section{AUTHOR CONTRIBUTIONS}

IG-C and FJF-R assisted with experiments, analyzed data, and co-wrote the manuscript. SAC, AT, MAA, and JP-P conducted behavioral experiments. ARZ and MKL assisted with experimental design, data interpretation, and writing of the manuscript. SDI conceived and directed the project, analyzed data, interpreted results, and wrote the manuscript. All authors reviewed and edited the manuscript.

\section{ADDITIONAL INFORMATION}

Supplementary Information accompanies this paper at (https://doi.org/10.1038/ s41386-020-0654-7).

Publisher's note Springer Nature remains neutral with regard to jurisdictional claims in published maps and institutional affiliations.

\section{REFERENCES}

1. Keyes KM, Gary D, O'Malley PM, Hamilton A, Schulenberg J. Recent increases in depressive symptoms among US adolescents: trends from 1991 to 2018. Soc Psychiatry Psychiatr Epidemiol. 2019;54:987-96.

2. Roy A, Oldehinkel AJ, Verhulst FC, Ormel J, Hartman CA. Anxiety and disruptive behavior mediate pathways from attention-deficit/hyperactivity disorder to depression. J Clin Psychiatry. 2014;75:e108-113.

3. Birmaher B, Ryan ND, Williamson DE, Brent DA, Kaufman J. Childhood and adolescent depression: a review of the past 10 years. Part II. J Am Acad Child Adolesc Psychiatry. 1996;35:1575-83.

4. Pezawas L, Wittchen HU, Pfister H, Angst J, Lieb R, Kasper S. Recurrent brief depressive disorder reinvestigated: a community sample of adolescents and young adults. Psychol Med. 2003;33:407-18.

5. Ordaz SJ, Goyer MS, Ho TC, Singh MK, Gotlib IH. Network basis of suicidal ideation in depressed adolescents. J Affect Disord. 2018;226:92-9.

6. Monteggia LM, Zarate C Jr. Antidepressant actions of ketamine: from molecular mechanisms to clinical practice. Curr Opin Neurobiol. 2015;30:139-43.

7. Zarate CA Jr., Singh JB, Carlson PJ, Brutsche NE, Ameli R, Luckenbaugh DA, et al. A randomized trial of an $\mathrm{N}$-methyl-D-aspartate antagonist in treatment-resistant major depression. Arch Gen Psychiatry. 2006;63:856-64.

8. Berman RM, Cappiello A, Anand A, Oren DA, Heninger GR, Charney DS, et al. Antidepressant effects of ketamine in depressed patients. Biol Psychiatry. 2000;47:351-4

9. Cullen KR, Amatya P, Roback MG, Albott CS, Westlund Schreiner M, Ren $Y$, et al. Intravenous ketamine for adolescents with treatment-resistant depression: an open-label study. J Child Adolesc Psychopharmacol. 2018;28:437-44.

10. Autry AE, Adachi M, Nosyreva E, Na ES, Los MF, Cheng PF, et al. NMDA receptor blockade at rest triggers rapid behavioural antidepressant responses. Nature. 2011;475:91-5. 
11. Murrough JW. Ketamine as a novel antidepressant: from synapse to behavior. Clin Pharmacol Ther. 2012;91:303-9.

12. Iñiguez SD, Flores-Ramirez FJ, Riggs LM, Alipio JB, Garcia-Carachure I, Hernandez $M A$, et al. Vicarious social defeat stress induces depression-related outcomes in female mice. Biol Psychiatry. 2018;83:9-71.

13. Li N, Lee B, Liu RJ, Banasr M, Dwyer JM, Iwata M, et al. mTOR-dependent synapse formation underlies the rapid antidepressant effects of NMDA antagonists. Science. 2010;329:959-64.

14. Popik P, Kos T, Sowa-Kucma M, Nowak G. Lack of persistent effects of ketamine in rodent models of depression. Psychopharmacology. 2008;198:421-30.

15. Morgan CJ, Curran HV. Ketamine use: a review. Addiction. 2012;107:27-38.

16. Zarrinnegar $P$, Kothari J, Cheng K. Successful use of ketamine for the treatment of psychotic depression in a teenager. J Child Adolesc Psychopharmacol. 2019;29:1-2.

17. Andersen SL. Stimulants and the developing brain. Trends Pharmacol Sci. 2005;26:237-43.

18. Doremus-Fitzwater TL, Varlinskaya El, Spear LP. Motivational systems in adolescence: possible implications for age differences in substance abuse and other risk-taking behaviors. Brain Cogn. 2010;72:114-23.

19. Flores-Ramirez FJ, Parise LF, Alipio JB, Garcia-Carachure I, Castillo SA, Rodriguez $M$, et al. Adolescent fluoxetine history impairs spatial memory in adult male, but not female, C57BL/6 mice. J Affect Disord. 2019;249:347-56.

20. Milstein JA, Elnabawi A, Vinish M, Swanson T, Enos JK, Bailey AM, et al. Olanzapine treatment of adolescent rats causes enduring specific memory impairments and alters cortical development and function. PLoS ONE 2013;8:e57308.

21. Izquierdo A, Pozos H, Torre Ade L, DeShields S, Cevallos J, Rodriguez J, et al. Sex differences, learning flexibility, and striatal dopamine D1 and D2 following adolescent drug exposure in rats. Behav Brain Res. 2016;308:104-14.

22. Vinish M, Elnabawi A, Milstein JA, Burke JS, Kallevang JK, Turek KC, et al. Olanzapine treatment of adolescent rats alters adult reward behaviour and nucleus accumbens function. Int J Neuropsychopharmacol. 2013;16:1599-609.

23. Halladay LR, Iñiguez SD, Furqan F, Previte MC, Chisum AM, Crawford CA. Methylphenidate potentiates morphine-induced antinociception, hyperthermia, and locomotor activity in young adult rats. Pharmacol Biochem Behav. 2009;92:190-6.

24. Rincón-Cortés M. Adolescent Prozac administration exerts antidepressant-like effects in adulthood by reducing ERK2 signaling in the VTA. J Neurosci. 2014;34:6719-20.

25. Flores-Ramirez FJ, Garcia-Carachure I, Sanchez DO, Gonzalez C, Castillo SA, Arenivar MA, et al. Fluoxetine exposure in adolescent and adult female mice decreases cocaine and sucrose preference later in life. J Psychopharmacol. 2018. https://doi.org/10.1177/0269881118805488.

26. Iñiguez SD, Riggs LM, Nieto SJ, Wright KN, Zamora NN, Cruz B, et al. Fluoxetine exposure during adolescence increases preference for cocaine in adulthood. Sci Rep. 2015;5:15009.

27. Alcantara LF, Warren BL, Parise EM, Iñiguez SD, Bolaños-Guzmán CA. Effects of psychotropic drugs on second messenger signaling and preference for nicotine in juvenile male mice. Psychopharmacology. 2014;231:1479-92.

28. Crawford CA, Baella SA, Farley CM, Herbert MS, Horn LR, Campbell RH, et al. Early methylphenidate exposure enhances cocaine self-administration but not cocaine-induced conditioned place preference in young adult rats. Psychopharmacology. 2011;213:43-52.

29. Carlezon WA Jr., Mague SD, Andersen SL. Enduring behavioral effects of early exposure to methylphenidate in rats. Biol Psychiatry. 2003;54:1330-7.

30. Xu S, Gullapalli RP, Frost DO. Olanzapine antipsychotic treatment of adolescent rats causes long term changes in glutamate and GABA levels in the nucleus accumbens. Schizophr Res. 2015;161:452-7.

31. Dahmani S, Michelet D, Abback PS, Wood C, Brasher C, Nivoche Y, et al. Ketamine for perioperative pain management in children: a meta-analysis of published studies. Paediatr Anaesth. 2011;21:636-52.

32. Forrester KR, Thomas SM, Gupta NK, Karumuri M, Gerard JM. Repeat intravenous ketamine dosing in children undergoing emergency department procedural sedation. J Emerg Med. 2019;56:1-6.

33. Papolos DF, Teicher MH, Faedda GL, Murphy P, Mattis S. Clinical experience using intranasal ketamine in the treatment of pediatric bipolar disorder/fear of harm phenotype. J Affect Disord. 2013;147:431-6.

34. Abdallah CG, Fasula M, Kelmendi B, Sanacora G, Ostroff R. Rapid antidepressant effect of ketamine in the electroconvulsive therapy setting. J Ect. 2012;28:157-61.

35. Liebrenz M, Stohler R, Borgeat A. Repeated intravenous ketamine therapy in a patient with treatment-resistant major depression. World J Biol Psychiatry. 2009;10:640-3.

36. Parise EM, Alcantara LF, Warren BL, Wright KN, Hadad R, Sial OK, et al. Repeated ketamine exposure induces an enduring resilient phenotype in adolescent and adult rats. Biol Psychiatry. 2013;74:750-9.

37. Kreibich AS, Briand L, Cleck JN, Ecke L, Rice KC, Blendy JA. Stress-induced potentiation of cocaine reward: a role for CRF R1 and CREB. Neuropsychopharmacology. 2009;34:2609-17.
38. Aguilar MA, Garcia-Pardo MP, Montagud-Romero S, Minarro J, Do Couto BR Impact of social stress in addiction to psychostimulants: what we know from animal models. Curr Pharm Des. 2013;19:7009-25.

39. Abreu-Villaca Y, Filgueiras CC, Guthierrez M, Medeiros AH, Mattos MA, Pereira Mdos S, et al. Exposure to tobacco smoke containing either high or low levels of nicotine during adolescence: differential effects on choline uptake in the cerebral cortex and hippocampus. Nicotine Tob Res. 2010;12:776-80.

40. Nugent AC, Ballard ED, Gould TD, Park LT, Moaddel R, Brutsche NE, et al. Ketamine has distinct electrophysiological and behavioral effects in depressed and healthy subjects. Mol Psychiatry. 2019;24:1040-52.

41. Dobry Y, Braquehais MD, Sher L. Bullying, psychiatric pathology and suicidal behavior. Int J Adolesc Med Health. 2013;25:295-9.

42. Solomon MB. Evaluating social defeat as a model for psychopathology in adult female rodents. J Neurosci Res. 2017;95:763-76.

43. Iñiguez SD, Warren BL, Neve RL, Russo SJ, Nestler EJ, Bolaños-Guzmán CA. Viralmediated expression of extracellular signal-regulated kinase- 2 in the ventral tegmental area modulates behavioral responses to cocaine. Behav Brain Res. 2010;214:460-4.

44. Bardo MT, Bevins RA. Conditioned place preference: what does it add to our preclinical understanding of drug reward? Psychopharmacology. 2000;153:31-43.

45. Carlezon WA Jr. Place conditioning to study drug reward and aversion. Methods Mol Med. 2003;84:243-9.

46. Wallace DL, Vialou V, Rios L, Carle-Florence TL, Chakravarty S, Kumar A, et al. The influence of DeltaFosB in the nucleus accumbens on natural reward-related behavior. J Neurosci. 2008;28:10272-7.

47. Iñiguez SD, Riggs LM, Nieto SJ, Dayrit G, Zamora NN, Shawhan KL, et al. Social defeat stress induces a depression-like phenotype in adolescent male c57BL/6 mice. Stress. 2014;17:247-55.

48. Warren BL, Vialou VF, Iñiguez SD, Alcantara LF, Wright KN, Feng J, et al. Neurobiological sequelae of witnessing stressful events in adult mice. Biol Psychiatry. 2013;73:7-14.

49. Iñiguez SD, Aubry A, Riggs LM, Alipio JB, Zanca RM, Flores-Ramirez FJ, et al. Social defeat stress induces depression-like behavior and alters spine morphology in the hippocampus of adolescent male C57BL/6 mice. Neurobiol Stress. 2016;5:54-64.

50. Donahue RJ, Muschamp JW, Russo SJ, Nestler EJ, Carlezon WA Jr. Effects of striatal DeltaFosB overexpression and ketamine on social defeat stress-induced anhedonia in mice. Biol Psychiatry. 2014;76:550-8.

51. Trujillo KA, Zamora JJ, Warmoth KP. Increased response to ketamine following treatment at long intervals: implications for intermittent use. Biol Psychiatry. 2008;63:178-83.

52. Bates MLS, Trujillo KA. Long-lasting effects of repeated ketamine administration in adult and adolescent rats. Behav Brain Res. 2019;369:111928.

53. Schak KM, Vande Voort JL, Johnson EK, Kung S, Leung JG, Rasmussen KG, et al Potential risks of poorly monitored ketamine use in depression treatment. Am J Psychiatry. 2016;173:215-8.

54. Tzschentke TM. Measuring reward with the conditioned place preference (CPP) paradigm: update of the last decade. Addiction Biol. 2007;12:227-462.

55. Warren BL, Sial OK, Alcantara LF, Greenwood MA, Brewer JS, Rozofsky JP, et al. Altered gene expression and spine density in nucleus accumbens of adolescent and adult male mice exposed to emotional and physical stress. Dev Neurosci. 2014;36:250-60.

56. Krishnan V, Han MH, Graham DL, Berton O, Renthal W, Russo SJ, et al. Molecular adaptations underlying susceptibility and resistance to social defeat in brain reward regions. Cell. 2007;131:391-404.

57. Warren BL, Mazei-Robison MS, Robison AJ, Iñiguez SD. Can I get a witness? Using vicarious defeat stress to study mood-related illnesses in traditionally understudied populations. Biol Psychiatry. 2020. https://doi.org/10.1016/j. biopsych.2020.02.004.

58. Burke AR, Miczek KA. Escalation of cocaine self-administration in adulthood after social defeat of adolescent rats: role of social experience and adaptive coping behavior. Psychopharmacology. 2015;232:3067-79.

59. Fosnocht AQ, Lucerne KE, Ellis AS, Olimpo NA, Briand LA. Adolescent social isolation increases cocaine seeking in male and female mice. Behav Brain Res. 2019;359:589-96.

60. Rodriguez-Arias M, Montagud-Romero S, Rubio-Araiz A, Aguilar MA, Martin-Garcia $E$, Cabrera R, et al. Effects of repeated social defeat on adolescent mice on cocaine-induced CPP and self-administration in adulthood: integrity of the bloodbrain barrier. Addict Biol. 2017;22:129-41.

61. Berton O, McClung CA, Dileone RJ, Krishnan V, Renthal W, Russo SJ, et al. Essential role of BDNF in the mesolimbic dopamine pathway in social defeat stress. Science. 2006;311:864-8.

62. Nakatake $Y$, Furuie $H$, Yamada M, Kuniishi H, Ukezono M, Yoshizawa K, et al. The effects of emotional stress are not identical to those of physical stress in mouse model of social defeat stress. Neurosci Res. 2019. https://doi.org/10.1016/j. neures.2019.10.008 
Enduring effects of adolescent ketamine exposure on cocaine- and...

I Garcia-Carachure et al.

1544

63. Hoeschel K, Guba K, Kleindienst N, Limberger MF, Schmahl C, Bohus M. Oligodipsia and dissociative experiences in borderline personality disorder. Acta Psychiatr Scand. 2008;117:390-3.

64. Nadler IM, Hariprasad MK. Psychogenic oligodipsia with hypernatremia in a psychotic patient. Am J Psychiatry. 1980;137:1269-70.

65. McDougall SA, Moran AE, Baum TJ, Apodaca MG, Real V. Effects of ketamine on the unconditioned and conditioned locomotor activity of preadolescent and adolescent rats: impact of age, sex, and drug dose. Psychopharmacology. 2017;234:2683-96.

66. Crawford CA, Moran AE, Baum TJ, Apodaca MG, Montejano NR, Park Gl, et al. Effects of monoamine depletion on the ketamine-induced locomotor activity of preweanling, adolescent, and adult rats: sex and age differences. Behav Brain Res. 2020;379:112267.

67. Kokkinou M, Ashok AH, Howes OD. The effects of ketamine on dopaminergic function: meta-analysis and review of the implications for neuropsychiatric disorders. Mol Psychiatry. 2018;23:59-69.

68. Cooper SJ, Francis J, Al-Naser H, Barber D. Evidence for dopamine D-1 receptormediated facilitatory and inhibitory effects on feeding behaviour in rats. J Psychopharmacol. 1992;6:27-33.

69. Shinohara F, Kamii H, Minami M, Kaneda K. The role of dopaminergic signaling in the medial prefrontal cortex for the expression of cocaine-induced conditioned place preference in rats. Biol Pharm Bull. 2017:40:1983-9.
70. Narendran R, Frankle WG, Keefe R, Gil R, Martinez D, Slifstein M, et al. Altered prefrontal dopaminergic function in chronic recreational ketamine users. Am J Psychiatry. 2005;162:2352-9.

71. Thelen C, Sens J, Mauch J, Pandit R, Pitychoutis PM. Repeated ketamine treatment induces sex-specific behavioral and neurochemical effects in mice. Behav Brain Res. 2016;312:305-12.

72. Yang C, Shirayama Y, Zhang JC, Ren Q, Yao W, Ma M, et al. R-ketamine: a rapidonset and sustained antidepressant without psychotomimetic side effects. Transl Psychiatry 2015;5:e632.

73. Duman RS, Li N. A neurotrophic hypothesis of depression: role of synaptogenesis in the actions of NMDA receptor antagonists. Philos Trans R Soc Lon B Biol Sci. 2012;367:2475-84.

74. Liu RJ, Lee FS, Li XY, Bambico F, Duman RS, Aghajanian GK. Brain-derived neurotrophic factor Val66Met allele impairs basal and ketamine-stimulated synaptogenesis in prefrontal cortex. Biol Psychiatry. 2012;71:996-1005.

75. Li X, Wolf ME. Multiple faces of BDNF in cocaine addiction. Behav Brain Res. 2015;279:240-54.

76. Hasegawa S, Yoshimi A, Mouri A, Uchida Y, Hida H, Mishina M, et al. Acute administration of ketamine attenuates the impairment of social behaviors induced by social defeat stress exposure as juveniles via activation of alphaamino-3-hydroxy-5-methyl-4-isoxazolepropionic acid (AMPA) receptors. Neuropharmacology. 2019;148:107-16. 